A. Pramesh Rao, G. Swarup and Gopal-Krishna, eds.

\title{
Radio Spectra of Complete Sample of Galactic Supernova Remnants
}

\author{
S.A. Trushkin
}

Special Astrophysical Observatory RAN, Nizhnij Arkhyz, Russia, 357147

\begin{abstract}
We present radio continuum spectra for nearly 200 Galactic supernova remnants (SNRs) from 220 known and included in Green's (1998) catalog. Spectra plotting is an "on-line" procedure of the CATS database http://cats. sao.ru/ and it could plot quite accurate spectra with the thermal plasma free-free absorption in fitting the spectra accounted for: to indicate the presence of extended ionized medium along the line of sight.

We did not find considerable correlation between spectral index and Galactic coordinates $l, b$ of SNRs. An analysis of 190 spectra showed that 78 SNRs (40\%) have clear low-frequency turnover caused, apparently, by absorption in the thermal foreground of the Milky Way. The turnover frequency $\nu_{\tau=1}$ for these SNRs has mean value near $30 \mathrm{MHz}$ do not correlate with the Galactic coordinates. But the frequency has significant correlation with the distance to SNR, defined from $\Sigma-D$ relation.
\end{abstract}

\section{Introduction}

The current catalog of SNRs (Green, 1998) contains 220 Galactic SNRs and some dozens of possible or probable ones. Reviews of radio spectra of a few dozen SNRs are given in many early papers and the recent papers by Kassim (1989a,b), Kovalenko et al. (1994). We measured the flux densities of nearly 120 SNRs (Trushkin, 1986, 1996ab, 1997, 1999; Trushkin et al., 1988) at several (up to six) frequencies, which corrected and complemented the spectra of many SNRs.

We collected all these data and make them accessible in the CATS database (Verkhodanov et al., 1997). The spectrum plotting procedure of the SNRs with optional fitting is designed to unify the statistical investigations of SNRs.

While there are no significant correlations of global SNR parameters with the spectral indices (Caswell and Clark, 1975; Lerche, 1980), Weiler (1983) proposed an extremely important classification which divided the SNRs into groups: shells, plerions, and composite or mixed SNRs. Accurate spectra are very important for the classification "shell/plerion", recognition of the mechanism of generation of relativistic particles, search for possible high- or low-frequency turnovers of spectra.

The theoretical calculations of evolution of radio spectra and possible correlation between spectral index and surface brightness (" $\Sigma-D$ ", " $\alpha-\Sigma$ " planes) 


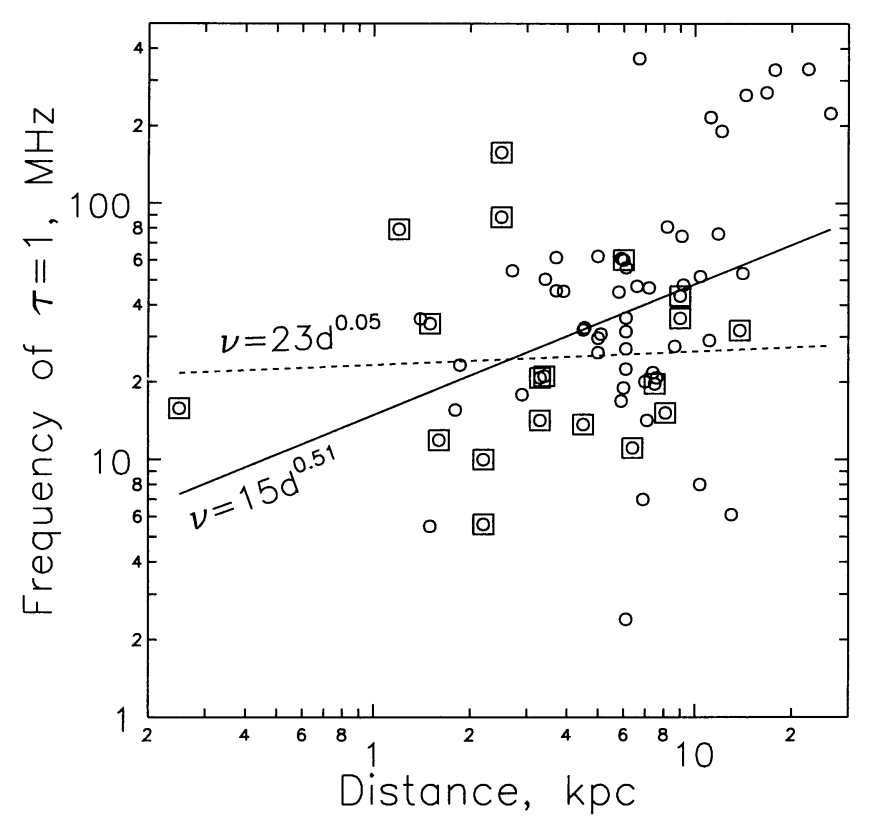

Figure 1. Turnover frequency $(\tau=1)$ for 70 SNRs via estimated distance $d_{\Sigma-D}$. The best fittings is given for total sample (marked by circles) and for sample of SNRs with known distances (marked by circles in squares).

should be confirmed by observational data of the total samples to draw conclusions concerning the physical evolution of SNRs.

\section{Analysis of Spectra}

The thermal absorption by the foreground well describes spectra of most SNRs:

$$
S_{\nu}=\left[S_{\nu_{0}}\left(\nu / \nu_{0}\right)^{\alpha}\right] \exp \left[-\tau_{\nu_{0}}\left(\nu / \nu_{0}\right)^{-2.1}\right] \text {, }
$$

Kassim (1989b) used the low-frequency data to derive spectra for 32 SNRs, and their turnovers at low frequencies $(<100 \mathrm{MHz})$ to indicate the presence of extended ionized medium along the line of sight.

Trushkin (1998) presented atlas of spectra of 200 SNRs: 192 SNRs, included in Green's catalog and the spectra of eight new SNRs or SNR candidates. The current statistics of the spectra includes the near 90 linear ones, 78 - linear with low frequency turnovers, 10 - with low frequency turn-ups, 3 - with high frequency turnovers for the SNRs in 220 Green's catalog (plus 10 new ones) 26 have only one measured flux density point ("no spectra"); 8 - two such points, 26 - three such points; other SNRs have more than four points.

There are few data for SNR with large angular size at frequencies higher than $10 \mathrm{GHz}$ in our catalog. Only in these SNRs with big ages such turnovers are due to synchrotron losses. Thus the high-frequency surveys of the SNRs are needed. 
The sample mean spectral indices at $4 \mathrm{GHz}$ are $\alpha_{4}=-0.50 \pm 0.21$. Spectral index do not correlate with the Galactic coordinates.

Bell's (1978a,b) mechanism (as a variant of the Fermi acceleration of the first order) of repowering is attractive because it provides a simple explanation of the observed spread of spectral indices from -0.5 to -1.1 : if the equation of state in SNR is described by strong adiabatic or isothermal shocks.

An analysis of 190 spectra showed that 78 SNRs (41\%) have clear lowfrequency turnover caused, apparently, by absorption in the thermal foreground of the Milky Way. These frequencies $\nu_{\tau=1}$ do not correlate with the Galactic coordinates, while $\nu_{\tau=1}$ in anticenter direction are below than in Galactic Center direction. The half of these SNRs have frequency of turnover $\left(\nu_{\tau=1}\right)$ below 40 $\mathrm{MHz}$.

The catalog of SNR spectra has ten cases of clear turn-up at low frequencies. Five such SNRs contain radio pulsars (Kaspi, 1998). There is significant correlation of the frequency $\nu_{\tau=1}$ and distances, estimated from $\Sigma-D$ relation $(\rho=0.61 \pm 0.05)$. It could be easily explained by either increasing the probability to catch at a line of sight the absorbing HII region or increasing of the path in absorbing warm ionized interstellar medium.

We could search for correlation between the turnover frequency and distance of SNRs. Because the distance $d$ estimates for individual SNRs are very uncertain, we could only use $\Sigma-D$ relation for estimate of distances to SNRs. A new refined $\Sigma-D$ relation was obtained by Case \& Bhattachatya (1998) for a sample of 36 Galactic shell SNRs:

$$
\Sigma_{1 \mathrm{GHz}}\left(\mathrm{W} \mathrm{Hz}^{-1} \mathrm{~m}^{-2} \mathrm{sr}^{-1}\right)=2.07_{-1.24}^{+3.10} \times 10^{-17} \mathrm{D}_{\mathrm{pc}}^{-2.38 \pm 0.26} .
$$

The thermal coefficient of absorption is defined:

$$
\tau_{\nu}=1.643 \cdot 10^{5} a\left(T_{e}, \nu\right) \cdot \nu^{-2.1} E M \cdot T_{e}^{-1.35} .
$$

If emission measure $E M=\int n_{e}^{2} d l \approx \overline{n_{e}^{2}} \cdot d$, then the condition $\tau_{\nu}=1$ gives:

$$
\left(\frac{\nu_{\tau=1}}{\mathrm{MHz}}\right)=21.9\left(\frac{T_{e}}{10^{4} \mathrm{~K}}\right)^{-0.64}\left(\frac{\bar{n}_{e}}{\mathrm{~cm}^{-3}}\right)^{0.95}\left(\frac{d}{\mathrm{kpc}}\right)^{0.48} .
$$

Thus using the best fitting of the $\nu_{\tau=1}-d$ dependence for 70 SNRs with low frequency turnovers we obtain:

$$
\left(\frac{T_{e}}{10^{4} \mathrm{~K}}\right)^{-0.64}\left(\frac{\bar{n}_{e}}{\mathrm{~cm}^{-3}}\right)^{0.95} \approx 0.7
$$

We could see from Figure 1 that a fitting of the unrefined $\nu_{\tau=1}-d$ dependence is similar to theoretical estimates from the thermal absorption formulae. The values: $T_{e}=7000 \mathrm{~K}$ and $\bar{n}_{e}=0.5 \mathrm{~cm}^{-3}$, typical parameters for warm ionized medium, well fit to the sample of the SRNs having low frequency turnovers.

From 16 SNRs with active neutron stars (Frail, 1998), the spectra of 15 ones have no low-frequency turnover at $>20-50 \mathrm{MHz}$ that could be related with the contribution of neutron stars (or pulsars) inside SNRs. 


\section{Conclusions}

We present radio continuum spectra for 192 from 220 known Galactic supernova remnants (SNRs). The catalog contains about 2300 flux density measurements. Where it was possible, the flux measurements were reduced to the common flux scale of Baars (1977), as it was done in the work of Kassim (1989a).

The procedure of spectrum plotting based on this catalog is "on-line" in the CATS data base http://cats.sao.ru/snr_spectra.html. These spectra include most flux density measurements from literature and our measurements of flux densities of nearly 120 SNRs with the RATAN-600 radio telescope.

There is significant correlation $(\rho=0.61)$ of the frequency $\nu_{\tau=1}$ and $d_{\Sigma-D}$, probably because absorption in warm ionized medium in the foreground in the SNR direction.

Acknowledgments. The author is grateful to his colleagues O.V. Verkhodanov, V.N. Chernenkov and H. Andernach who created the CATS database and to the Russian Foundation for Basic Research for financial support of the CATS project (grant No 96-07-89075).

\section{References}

Baars, J.W.M., Genzel, R., Pauliny-Toth, I.I.K., et. al. 1977, A\&A, 61, 99 Bell, A.R. 1978a, MNRAS, 182, 147

Bell, A.R.: 1978b, MNRAS, 182, 443

Case, G. L., \& Bhattacharya, D. 1998, ApJ, 504, 761

Clark, D.H., \& Caswell, J.L. 1976, MNRAS, 174, 267

Green, D.A. 1998, A Catalogue of Galactic Supernova Remnants (1998 September version), MRAO, UK (available on the WWW at "http://www.mrao.cam.ac.uk/surveys/snrs/").

Kaspi, V.M. 1998, Advances in Space Research, 21, 167

Kassim, N.E. 1989a, ApJ, 347, 915

Kassim, N.E. 1989b, ApJS, 71, 799

Kovalenko, A.V., Pynzar', A.V., \& Udal'tsov, V.A. 1994, Astron. Rep., 38, 95

Lerche, I. 1980, A\&A, 85, 141

Trushkin, S.A. 1986, Astron. Tsirk., 1453, 4

Trushkin, S.A., Vitkovskij V.V., \& Nizhelskij N.A. 1988, Astrofiz. Issled. (Izv. $\mathrm{SAO}), 25,84$

Trushkin, S.A. 1996a, Bull. SAO, 41, 64

Trushkin, S.A. 1996b, Astron. Astrophys. Trans., 11, 225

Trushkin, S.A. 1998, Bull. SAO, 46, 65

Trushkin, S.A. 1999, A\&A, 352, L103

Verkhodanov, O.V., Trushkin, S.A., Andernach, H., \& Chernenkov, V.N. 1997, Astronomical Data Analysis Software Systems VI, eds. G.Hunt \&

H.E.Payne. ASP Conf. Series, 125, 322

Weiler K.W. 1983, Observatory, 1054, 85 